Meta

Journal des traducteurs

Translators' Journal

\title{
Pourquoi enseigner l'interprétation simultanée avec texte en anglais - cas d'étude entre le français et le coréen
}

\section{Hyéwon Pyoun}

Volume 51, numéro 2, juin 2006

Théories et pratiques de la traduction et de l'interprétation en Corée Theories and Practices of Translation and Interpretation in Korea

URI : https://id.erudit.org/iderudit/013255ar

DOI : https://doi.org/10.7202/013255ar

Aller au sommaire du numéro

Éditeur(s)

Les Presses de l'Université de Montréal

ISSN

0026-0452 (imprimé)

1492-1421 (numérique)

Découvrir la revue

Citer cet article

Pyoun, H. (2006). Pourquoi enseigner l'interprétation simultanée avec texte en anglais - cas d'étude entre le français et le coréen. Meta, 51(2), 263-272.

https://doi.org/10.7202/013255ar
Résumé de l'article

De plus en plus d'orateurs utilisent comme support des documents en anglais tout en présentant leurs discours dans leur langue natale lors de conférences internationales. Cette situation mène les interprètes coréens-français à effectuer des interprétations simultanées avec texte à partir des supports visuels anglais. Deux canaux, l'un auditif et l'autre visuel, interviennent entre trois langues, coréen, français et anglais, pour accroître la charge mentale de l'interprète qui n'a pas l'anglais dans sa combinaison linguistique. Toutefois, souvent pratiquée dans le contexte coréen, l'interprétation simultanée faisant intervenir un support visuel anglais est négligée dans la formation de l'interprétation. Cet article a pour but d'analyser les erreurs commises par les étudiants en cours de formation afin de mettre en lumière la nécessité d’un enseignement de l'interprétation simultanée avec texte en anglais.
Ce document est protégé par la loi sur le droit d'auteur. L'utilisation des services d’Érudit (y compris la reproduction) est assujettie à sa politique d'utilisation que vous pouvez consulter en ligne.

https://apropos.erudit.org/fr/usagers/politique-dutilisation/ 


\title{
Pourquoi enseigner l'interprétation simultanée avec texte en anglais - cas d'étude entre le français et le coréen
}

\author{
HYÉWON PYOUN \\ Hankuk University of Foreign Studies, Seoul, Korea \\ hwpyoun@kornet.net
}

\section{RÉSUMÉ}

De plus en plus d'orateurs utilisent comme support des documents en anglais tout en présentant leurs discours dans leur langue natale lors de conférences internationales. Cette situation mène les interprètes coréens-français à effectuer des interprétations simultanées avec texte à partir des supports visuels anglais. Deux canaux, l'un auditif et l'autre visuel, interviennent entre trois langues, coréen, français et anglais, pour accroître la charge mentale de l'interprète qui n'a pas l'anglais dans sa combinaison linguistique. Toutefois, souvent pratiquée dans le contexte coréen, l'interprétation simultanée faisant intervenir un support visuel anglais est négligée dans la formation de l'interprétation. Cet article a pour but d'analyser les erreurs commises par les étudiants en cours de formation afin de mettre en lumière la nécessité d'un enseignement de l'interprétation simultanée avec texte en anglais.

\begin{abstract}
In conference settings that have Korean and French as official languages, more and more speakers prepare documents in English, while still speaking in either Korean or French. For Korean interpreters working at such conferences, the result is that they must perform simultaneous interpretation between Korean and French while referring to texts that are written in English. Two information streams - one oral and one visual - interact in three languages - Korean, French and English - to constitute quite extreme conditions of simultaneity and thus increases the interpreter's mental processing load. Furthermore, when an interpreter refers to an English text while listening to Korean discourse, s/he may be tempted to lean on the written text because of the syntactic similarities between English and French. This increases the risk of language interference between French - the interpreter's B language - and English, which is often not even the interpreter's C language. Though this is a problem that frequently arises in professional settings, simultaneous interpretation with English text is not dealt with in interpreter training programs. This paper utilizes an experiment with students in an interpreter training course to examine the needs associated with teaching this type of interpretation.
\end{abstract}

\section{초록}

점차 많은 국제회의에서 한국측 혹은 프랑스측 연사들은 영어로 된 자료를 바탕으 로 자신의 모국어로 발화하면서 한불통역사들은 영어자료를 바탕으로 한불간 텍스 트 동시를 수행하게 된다. 결국 통역사는 귀로는 연사의 한국어 혹은 불어 연설을 들으면서 눈으로는 영어 자료를 읽는 동시에 한국어 혹은 불어로 통역해야 하는 삼 중 과정을 처리해야 한다. 통역현장에서 이와 같은 상황이 빈번하게 발생함에도 불 구하고 교육현장에서는 텍스트 동시에 관한 수업조차 개설되어 있지 못한 형편이 다. 본고에서는 학생들을 대상으로 한 실험을 중심으로 영어 자료를 기본으로 하는 텍스트동시 교육의 필요성에 대해 살펴보고자 한다.

\section{MOTS-CLÉS/KEYWORDS}

interprétation simultanée avec texte, formation des interprètes, support visuel, analyse d'erreurs 


\section{Introduction}

Avant d'aborder la question relative à la nécessité d'enseigner l'interprétation simultanée avec texte en anglais, il convient tout d'abord d'expliquer ce que signifie l'expression. Elle présuppose avant tout une interprétation simultanée se faisant avec un texte qui n'est ni écrit dans la langue d'arrivée ni écrit dans la langue de départ de l'interprète. Cette langue est en l'occurence l'anglais en Corée et force est de constater que la plupart des interprètes professionnels coréens spécialisés dans deux langues autres que l'anglais n'ont malheureusement pas cette langue dans leur combinaison linguistique.

Ce type d'interprétation présuppose également que l'interprète, pris sous le feu des deux canaux - auditif et visuel -, doit maîtriser en même temps deux langues de départ, par exemple le coréen ou le français pour la partie auditive et l'anglais pour la partie texte. Dans la plupart des cas, les orateurs, qu'ils soient francophones ou coréanophones, optent pour une présentation de leurs écrits en anglais tout en prenant la parole dans leur langue maternelle. Cette situation survient lors des interprétations de relais, très fréquentes en Corée étant donné qu'une conférence trilingue nécessite systématiquement ce type d'interprétation. Et lorsqu'il y a recours au texte anglais, l'interprète écoute depuis la cabine pivot réservée au coréen en suivant ce texte pour l'interpréter ensuite, par exemple, en français.

Réfléchir sur les raisons pour lesquelles l'interprétation simultanée peut se faire via un texte en anglais mérite une attention particulière. Trois éléments fondent en tout cas l'importance de se pencher sur le sujet, encore largement inexploré.

Le premier revêt un aspect théorique. Aucune étude poussée n'a été consacrée jusqu'à présent aux effets d'une troisième langue intervenant dans le processus de l'interprétation simultanée. Li Feng (2001) a traité ce thème partiellement pour uniquement évoquer les difficultés nées de l'absence de documents dans la langue de travail des interprètes chinois. Ce point est certes non négligeable. Toutefois, des recherches approfondies en ce domaine pourraient mettre en lumière les stratégies adoptées par les interprètes professionnels lorsqu'ils font face à deux langues de départ dans les conditions extrêmes que sont celles de la simultanéité.

Le deuxième élément revêt un aspect pratique. De plus en plus d'orateurs préfèrent de loin que leurs textes anglais soient visualisés sur un écran. Aujourd'hui, les supports rédigés dans cette langue sont légions lors des conférences économiques et scientifiques. Cette situation concerne d'autres combinaisons linguistiques et celle voyant l'emploi de l'anglais est appelée à prendre de l'ampleur à l'avenir, cette langue étant devenue universelle. Nombreux interprètes coréens interrogés lors de séminaires internationaux admettent être en difficulté lorsque l'interprétation simultanée se fait avec un texte en anglais, sans toutefois pouvoir en expliquer les raisons ou proposer des stratégies adéquates pour se tirer d'affaire. Une analyse de corpus serait souhaitable en vue de trouver des solutions et d'élaborer un modèle en matière de traitement des deux canaux pour deux langues données et concernant les entrants.

Le troisième élément concerne l'aspect pédagogique. Alors que l'enseignement de l'interprétation a toujours été dispensé en fonction des aléas du terrain, on observe une disparité dans le domaine de l'interprétation simultanée ayant pour base un texte. Dans le curriculum actuel de notre école, il n'existe d'ailleurs aucun cours consacré à ce type d'interprétation, si bien que chaque enseignant se voit libre d'aborder le sujet lorsqu'il le désire. Il va sans dire que le travail via des textes anglais est pratiquement 
inexistant et que nos étudiants n'ont guère l'occasion, par conséquent, d'apprendre le métier dans les conditions réelles du terrain.

Nous nous proposerons de laisser de côté les aspects théorique et pratique et de nous occuper uniquement de la question pédagogique. Cet article sera donc concentré sur l'importance de pratiquer dans les conditions réelles du terrain, avec pour cadre une expérimentation à laquelle ont participé des étudiants de deuxième année.

\section{Expérimentation}

Les étudiants concernés par cette expérimentation étaient ceux de deuxième année de la section interprétation de conférence ${ }^{1}$. Ayant enseigné l'interprétation consécutive pendant un an à ces mêmes étudiants alors qu'ils étaient en première année, puis l'interprétation simultanée spécialisée ${ }^{2}$ pendant le premier semestre de leur deuxième année, nous avions conscience du niveau de chaque étudiant. Le nombre de participants a été de quatre, nombre relativement faible mais qui est celui de l'effectif total de la section interprétation de conférence. Parmi les quatre, deux avaient vécu en France plus de 8 ans - l'un deux connaît d'ailleurs le français comme sa propre langue maternelle - un, 6 ans et un autre, 3 ans. Comparée à celles des années précédentes, cette classe avait aussi une expérience de séjours plus longs à l'étranger, si bien que la maîtrise de la langue B leur était plus aisée par rapport à d'autres étudiants ayant eu à leur actif une seule année de séjour linguistique en France.

Les documents utilisés avaient récemment servi lors d'une conférence internationale portant sur les programmes mis en ouvre pour éliminer les accidents de la route. Les textes en format powerpoint étaient en anglais et le discours se basant sur ce support d'une durée de 10 minutes pour chaque intervention, c'est-à-dire 10 minutes depuis le coréen vers le français et 10 minutes depuis le français vers le coréen.

L'expérimentation a eu lieu en fin de premier semestre. Les documents powerpoint ${ }^{3}$ rédigés en anglais ont été distribués 20 minutes à l'avance et le thème de la conférence simulée a été donné une semaine auparavant. Les étudiants ont donc eu une huitaine de jours pour préparer un lexique sur le thème abordé, lexique qu'ils devaient rendre le jour de l'examen. Ils avaient eu trois cours d'interprétation simultanée pendant tout le semestre, mais c'était la première fois qu'ils utilisaient des powerpoints en anglais.

Les étudiants éprouvent tous des difficultés lors de l'interprétation simultanée avec texte. Gile (1995a: 185) cite trois raisons pour expliquer ceci: les caractéristiques linguistiques des textes écrits différenciant ceux-ci des discours, les risques d'interférence linguistique et la difficulté de suivre le discours oral en même temps que le texte écrit. Dans sa thèse de doctorat, Déjean (1978) souligne également les difficultés liées à la lecture du texte par rapport à une situation d'improvisation orale. Toutefois, lors de notre test, il n'a pas été tenu compte des caractéristiques linguistiques des textes écrits qui ont été des powerpoints, étant donné que ces derniers en montrent peu. C'est la raison d'ailleurs pour laquelle ils ont été choisis, car une lecture d'un texte entièrement rédigé aurait signifié une tâche trop difficile pour les étudiants. Dans le cas des powerpoints, les étudiants ont pu bénéficier d'une marge de manœuvre suffisante pour contrôler leurs productions. Cette expérimentation aura de toute façon été un point de départ pour une recherche approfondie dans le domaine de l'interprétation simultanée voyant la mise en jeu d'un texte-support en anglais. 
Une première difficulté ayant été éliminée, il a fallu nous intéresser à une autre. Les risques d'interférence linguistique restent toujours importants car l'interprète doit, dans certaines situations, se référer au texte anglais, comme c'est souvent le cas depuis l'anglais vers le français. En fait, il s'agit là d'un double problème, car l'interprète traduit vers la langue $B$ via une autre langue qui n'est même pas sa langue $C$. Si son intuition linguistique est réduite, il réagira de manière moins sensible aux interférences. Celles-ci prêtent en outre facilement à confusion, vu les maintes similarités syntaxiques $^{4}$ existant entre l'anglais et le français. Et c'est sous la pression que les étudiants en formation sont souvent tentés de recourir au texte anglais, ce qui rend plus facile le transcodage. La formule latine Verba volant, scripta manent pourrait suffire à elle-seule pour expliquer cette tendance émanant de la part des interprètes à vouloir s'appuyer davantage sur l'écrit que sur l'oral. Les similarités syntaxiques entre l'anglais et le français sont finalement une donnée supplémentaire.

La situation de simultanéité liée à l'utilisation de trois langues en même temps étant une réalité en Corée, l'interprète se voit régulièrement dans l'obligation de manipuler deux supports et de comparer sans cesse le premier - le support auditif coréen - au deuxième qui, lui, est un support visuel rédigé en anglais. Ainsi lui est-il plus difficile de se repérer lorsqu'il y a déviation de l'orateur par rapport au texte écrit.

\section{Analyse des résultats}

Le but de cette expérimentation était de vérifier les effets de l'utilisation de l'anglais et de ceux d'un support écrit, dans le domaine de l'interprétation simultanée. En cas d'effets négatifs, la production des étudiants en formation montrerait ainsi plus de transcodage en raison des interférences linguistiques et ces derniers seraient alors plus tentés d'interpréter le texte écrit au risque de se déconnecter du discours de l'orateur.

\subsection{Modalités de présentation de texte}

Maints auteurs ont souligné l'importance des modalités de présentation de texte à la suite de l'observation d'interprétations simultanées (Pöchhacker, 1994; Messina, 1998; Gile 1995b). La construction du corpus passe d'abord par la précision des variables émanant de l'entrant et parmi elles, le mode de présentation est de loin celle qui est la plus facilement contrôlable. Il n'en demeure pas moins que cet aspect a été peu étudié en tant que variable isolée.

Reprenant la classification de Kopczinski (cité dans Ondelli, 1998), Pöchhacker (1994: 237) élabore le text delivery profile et aborde la variable en fonction de la préparation du texte. Pour lui, un texte est extemporaneous, preconceived (notes ou plan sans texte écrit), presented (texte écrit destiné à être lu oralement) ou read (texte écrit destiné à être publié). L'interprétation simultanée avec texte met en jeu les deux derniers types de présentation, de loin les plus courants lors des conférences internationales - la fréquence d'apparition du texte anglais fera d'ailleurs l'objet d'une enquête ultérieure. Pour revenir aux powerpoints, l'orateur lit certaines parties écrites et improvise pour ce qui est du non-écrit.

C'est justement dans le traitement des parties écrites qu'interviennent les interférences linguistiques en raison de la présence de la troisième langue qu'est l'anglais. 
Celles qui surviennent entre la langue B et l'anglais peuvent avoir des effets encore plus désastreux car le risque de ne pas les reconnaître (Newmark, 1982: 162) augmente considérablement. Dans le contexte des pays non anglophones, dont fait partie la Corée, le text delivery profile doit prendre en considération la langue de présentation dans laquelle sont rédigés les documents lorsqu'elle est différente de la langue parlée par les orateurs.

\subsection{Analyse des erreurs}

L'analyse des interprétations a montré une différence par rapport à celles produites tout au long du semestre. Au lieu d'une transcription détaillée sur les erreurs repérées lors des interprétations qui ont eu lieu avec texte, nous ne citerons que les grandes catégories pour les plus fréquentes lorsque l'on compare aux interprétations sans texte, afin d'accentuer la nécessité de formation de l'interprétation simultanée ayant pour support un texte anglais.

Premièrement, nous observons plus d'omissions. Contrairement à des idées reçues, les textes, au lieu d'être une source d'information importante pouvant aider à remédier aux problèmes liés à la mémoire, constituent un obstacle pour les étudiants dont la charge mentale s'accroît, étant donné que les efforts fournis pour lire l'anglais viennent troubler leur écoute. L'exemple suivant, depuis un corpus coréen, illustre bien le genre d'omissions concernées ainsi que le manque de ciblage dans le processus d'interprétation.

$\mathrm{S}^{5}$ : 문제점은 교통사고 줄이기가 정착되었다는 판단아래 건교부로 사고 줄이기 업무를 이관했다는 점입니다. 1998년 교통사고 사망자수는 전년대비 $21.9 \%$ 감 소했습니다. 성공요인은 다음과 같습니다. IMF 경제위기로 교통량이 대폭 감소 했다는 것입니다.

(Convaincu que les accidents de route sont en baisse, le gouvernement transfere cette fonction sous le ministère des Transports et de la Construction ${ }^{6}$. En 1998, le nombre de tués sur les routes a baissé de 21,9\% par rapport à l'année précédente. Les facteurs de succès sont les suivants. La circulation a considérablement diminué en raison de la crise financière du pays.)

T: titre: 2nd Reduction (1998): $-21,9 \%$

Success factors: Traffic volume reduction due to economic crisis.

I: Cette fonction a donc été transférée au ministère des Transports. Et cela a entraîné une hausse des accidents de la route. (pause) Les causes sont les suivantes. La circulation a diminué à cause de la crise financière.

Après avoir éliminé le sous-titre de cette diapositive, l'étudiant ne se rend pas compte qu'il lie la hausse des accidents de la route à la diminution du trafic routier, laquelle explique pourtant la réduction des accidents. Une pause intervient alors car il essaie de repérer ce qu'il entend avec le texte et quand il perçoit que l'orateur parle de la diapositive suivante, il est déjà en retard par rapport à celui-ci. Sans se souvenir de ce qu'il vient de dire, il enchaîne directement avec les facteurs de réussite.

Ce qui est intéressant, c'est que tous les participants omettent des éléments essentiels, tels que les titres, puisqu'ils leur faut du temps pour se réorienter lorsque l'on passe à une nouvelle diapositive. En outre, le fait d'avoir sous les yeux le titre de chaque paragraphe conduit les étudiants à oublier le non-dit et souvent ils ont l'impression que le public partage ce qu'ils lisent concernant le texte, même s'ils ne le traduisent pas. 
Deuxièmement, nous observons plus de substitutions. Celui qui n'a pas saisi ce qui a été dit par l'orateur préfère lire le texte mis en jeu plutôt que de s'interrompre.

S: Après la Seconde Guerre mondiale jusqu'au début des années 1960, la Prévention routière est pratiquement la seule organisation à être connue et reconnue par l'opinion publique en matière de sécurité routière.

T1: 1949 - Prévention routière.

I 1: 도로안전국은 1949년에 창설되었습니다. (La Prévention routière a été fondée en 1949.)

Ainsi, sans tenir compte du discours oral, l'étudiant traduit le powerpoint. Souvent des orateurs ne mentionnent pas ce qui est écrit et ajoutent des informations supplémentaires. Dans ce cas, leur vouloir-dire n'est aucunement respecté.

Troisièmement, nous observons plus de transcodages. Alors que les interférences sont moindres entre le français et le coréen en raison de la quasi impossibilité du transcodage, la tentation est forte pour les étudiants faisant face à l'anglais. L'exemple suivant illustre le phénomène:

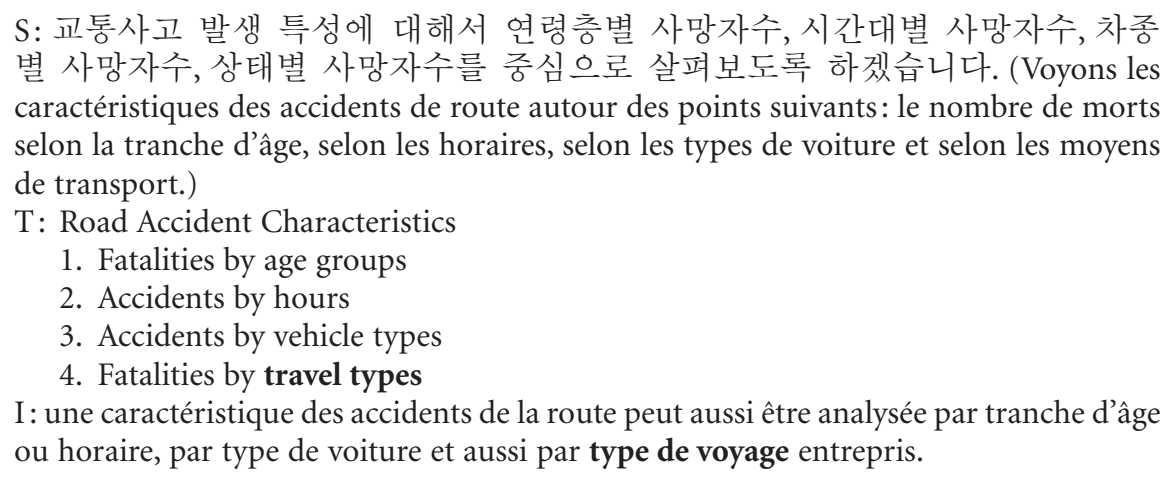

Que veut dire type de voyage? Alors que sur la diapositive figure un graphique sur lequel on peut lire piétons, bicyclettes, 2 roues, voitures, l'étudiant, oubliant ce qu'il a appris préalablement, transcode automatiquement travel type par type de voyage. Le transcodage du coréen donnerait type d'état car dans cette langue travel n'y figure pas. Dès que commence l'énumération, l'élève se déconnecte du texte pour un transcodage de l'anglais, opération qui lui semble plus facile.

Quatrièmement, nous observons plus de fautes grammaticales. Alors que leur niveau de français était plutôt relevé, les apprenants ont commis de nombreuses fautes de grammaire, fautes qui n'apparaissaient pas aussi fréquemment lors de leurs interprétations simultanées libres vers le français. La charge mentale supplémentaire, issue du fait de suivre le texte en même temps, détruit l'équilibre de l'étudiant qui sacrifie sa production en faveur de l'analyse (Gile, 1985). En effet, l'effort de compréhension devient plus grand lors du couplage des supports auditif et visuel.

\subsection{Modèle de processus d'interprétation simultanée avec texte}

Le processus d'interprétation simultanée avec texte en anglais peut être illustré comme suit, d'après le modèle de Weber (1990): 
Figure 1

Modèle de processus de la simultanée avec texte en anglais

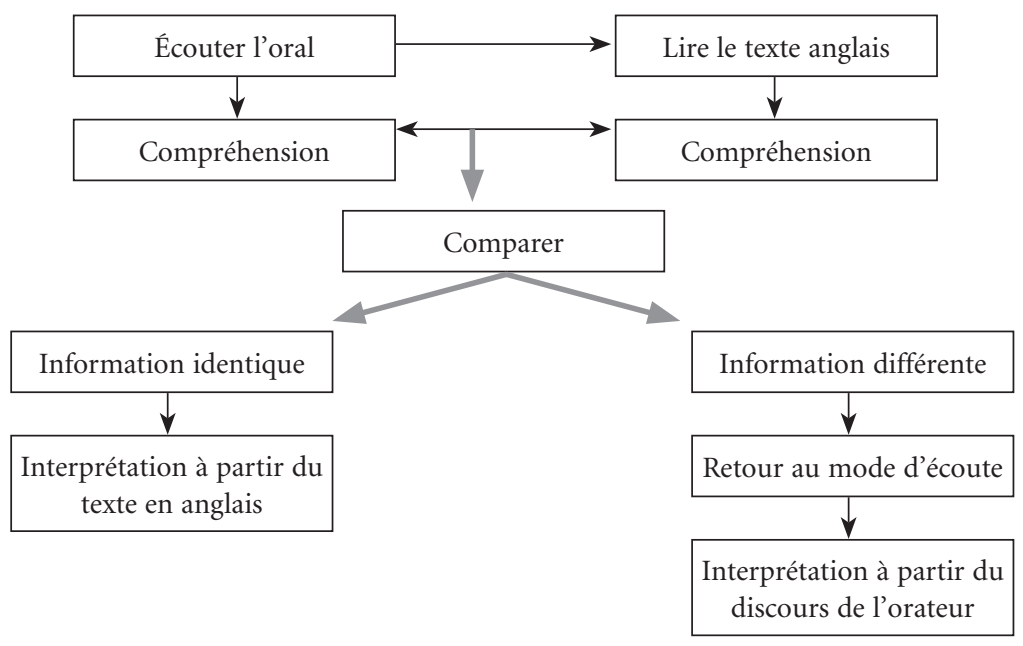

Les flèches en gras indiquent une charge mentale importante par rapport à l'exploitation de textes faite dans la langue de travail, car si la langue du texte et celle de l'orateur étaient la même, le repérage du passage serait automatique. Le premier mot énoncé par l'orateur suffira dans certains cas pour retrouver la phrase correspondante. Toutefois, dans le cas d'un texte anglais, des efforts supplémentaires devront être consentis car l'interprète aura à lire la phrase entière pour vérifier si elle correspond bien au passage mentionné par l'orateur. Souvent, le premier groupe de mots ne constituera pas un indice suffisant dans la recherche du passage correspondant. À cause de la présence du texte, l'interprète devra ainsi fournir un double effort pour la compréhension de l'énoncé et de celle du texte anglais.

En psycholinguistique, il a été dit que les yeux du lecteur qui lit à haute voix fixent 5 ou 7 mots à l'avance avant qu'il ne les lise (Huey, cité par Carroll, 2004). Le mouvement des yeux de celui qui suit la lecture sur support écrit suit le même rythme. Dans le cas de l'interprète faisant face à la lecture de l'orateur, il en est de même. Tout en concentrant son attention sur la partie lue par l'orateur, ce dernier fixe en même temps la partie suivante, soit un triple processus mental s'articulant sur l'audition du discours prononcé par l'orateur, le repérage lié à la compréhension du texte anglais et la saisie de la partie suivante.

\section{Retombées pédagogiques}

Ceci démontre bien la nécessité d'entraîner les étudiants à la pratique de l'interprétation simultanée avec texte en anglais, dans le cas de la Corée où une langue intermédiaire est un must, le coréen n'étant pas une langue universellement parlée. Seleskovitch (1989), Weber (1990) et Laplace (1998) ont souligné l'importance de la formation concernant la traduction à vue. La maîtrise des techniques des interprétations consécutive et simultanée ne suffit pas et l'interprète débutant ressent des difficultés lors d'une interprétation simultanée avec texte, particulièrement lorsqu'il n'a pas eu la possibilité de lire ce dernier, ce qui l'oblige ainsi à s'investir plus au niveau 
de l'écoute au risque d'omettre des informations qui figurent pourtant dans le texte. Un enseignement adéquat dispensé pendant la formation permettra de l'armer plus efficacement face à ce genre de situation.

Toutefois, la situation coréenne actuelle montre qu'il n'existe pas de cours spécialement consacré à l'interprétation simultanée avec texte. Certains enseignants, conscients du changement survenant sur le terrain, consacrent quelques heures sur le sujet dans le cadre de leurs cours semestriels. Conscients des difficultés soulevées, nous avons consacré le semestre de l'interprétation simultanée spécialisée sur le thème avec l'utilisation de powerpoints en anglais, quand les circonstances l'ont permis. Deux étudiants qui avaient dans leur combinaison l'anglais faisait partie du cours en question pendant le second semestre, l'exercice a été possible, si bien que le semestre de formation s'est révélé fructueux quant à surmonter les difficultés liées à l'emploi du support visuel.

Néanmoins, une interprétation simultanée avec texte rédigé en anglais semblait difficile à appliquer en classe et ce pour deux raisons. Premièrement, l'option devant être proposée que si l'on maîtrise les techniques de l'interprétation simultanée, elle ne pouvait par conséquent qu'être enseignée au cours du deuxième semestre de la deuxième année. Toutefois, l'importance de l'examen final est tel qu'il est pratiquement impossible de l' intégrer dans le curriculum. En effet, étant donné que la simultanée avec texte ne figure pas dans l'examen final, les étudiants ne sont pas prêts à consacrer leur deuxième semestre à une méthode d'interprétation qu'ils affronteraient seulement sur le terrain. Deuxièmement, dans l'état actuel où prévaut une combinaison majeure de deux langues, ni l'enseignant ni l'étudiant ne sont en mesure de préparer comme il le faudrait un texte anglais pour une production en simultané avec texte.

Quelles alternatives sont alors envisageables? Comme le souligne Weber (1990), il est important d'intégrer l'interprétation simultanée avec texte, même si celui-ci n'est pas en anglais, comme composante de l'examen final, ce qui accentue sa légitimité d'enseignement. D'un autre côté, un cours entièrement consacré à cette matière permettra à l'enseignant de proposer une sélection d'exercices depuis un texte anglais.

Toutefois, le problème de la maîtrise du texte anglais dresse un nouvel obstacle. Notre idée est de mener un cours conjointement avec la classe des étudiants qui ont l'anglais dans leur combinaison linguistique. Les étudiants coréen-français pourraient se joindre à eux pendant le cours d'interprétation simultanée avec texte afin d'être mieux préparés. Mais une difficulté en cache une autre, celle du relais. Les étudiants coréen-français devront toujours s'appuyer sur la cabine pivot lorsqu'ils interprèteront depuis le coréen vers le français, via un texte anglais.

Une autre solution serait l'élaboration d'un discours en coréen ou en français à partir d'un texte originalement anglais. L'utilisation d'un support, écrit par un anglophone, est une réponse à la difficulté de rédiger un texte dans cette langue. Mais le discours prononcé par un étudiant se basant sur ce texte pourra montrer les mauvais aspects liés à la traduction à vue. Pour que les étudiants n'aient pas l'impression d'être confrontés à une traduction à vue mal avancée, l'orateur devra rédiger son texte, destiné à être lu, avant son intervention. Un cours triangulaire impliquant la présence d'un professeur anglophone sera l'occasion d'une étude instructive des fautes produites dans la langue source lorsque celle-ci est le coréen. En ce qui concerne une production en simultané avec texte depuis le français vers le coréen, le cours triangulaire 
permettra au professeur de vérifier la fidélité de l'interprétation des étudiants. La pratique avec des textes en anglais permettra alors aux étudiants de prendre contact avec le terrain.

\section{Conclusion}

De plus en plus de documents anglais sont présentés lors des conférences internationales ou franco-coréennes. La fréquente projection de diapositives de powerpoint impose de montrer des textes rédigés en anglais pour que chaque partie puisse en saisir le sens. Souvent, les interprètes se voient contraints de pratiquer l'interprétation simultanée avec texte, mais en anglais. L'implication d'une troisième langue dans les conditions extrêmes que sont celles de la simultanéité entraîne inévitablement une charge mentale supplémentaire dans tout processus d'analyse de l'information de la part de l'entrant. Dans notre enseignement actuel, les interprètes affrontent ce phénomène pour la première fois lorsqu'ils font leurs premières armes sur le terrain.

L'expérimentation réalisée auprès d'étudiants en cours de formation a montré des erreurs typiques issues de l'utilisation d'un texte anglais. Des cours consacrés au type d'activité concernée seront les bienvenus pour mieux préparer les étudiants à la vie professionnelle. La mise en place de ces nouveaux cours ne se fera pas, en tout cas, sans celle d'un examen final pour évaluer si les étudiants ont pleinement pris conscience de l'importance de l'interprétation simultanée avec texte. Pour les non-anglophones, la maîtrise de l'anglais devra cependant être une réalité, tant dans l'acquisition des connaissances thématiques et terminologiques - étant donné que les documents distribués lors des conférences internationales sont pratiquement tous en anglais que dans celle des techniques mises en ouvre, au niveau de la pratique.

\section{NOTES}

1. À la GSIT, un examen d'aptitude a lieu en fin de première année et seuls ceux ayant passé cet examen sont admis à la section Interprétation de conférence. Les autres sont automatiquement admis à la section Traduction.

2. Dans la section Interprétation de conférence sont proposés trois cours d'interprétation simultanée: l'interprétation simultanée vers la langue A, l'interprétation simultanée vers la langue B et l'interprétation simultanée spécialisée qui couvre les deux sens.

3. Bien que les documents aient été des powerpoints, ils étaient plus des textes que des photos ou des graphiques. Pour l'analyse des erreurs, nous avons surtout pris en considération les erreurs produites dans la partie des textes que dans la partie improvisée afin de dégager l'influence de l'interprétation simultanée avec texte.

4. Le terme similarités est utilisé ici dans un sens relatif. En effet par rapport au français (SVO) et le coréen (SOV), l'anglais (SVO) s'approche plus du français.

5. S signifie le discours original, T le texte écrit et I l'interprétation.

6. Les passages en italique concernent la diapositive précédente.

\section{RÉFÉRENCES}

Caroll, D. W. (2004) : Psychology of Language, New York, Brooks/Cole Publishing.

DéJEAN Le FéAL, K. (1978): Lectures et improvisations-Incidences de la forme de l'énonciation sur la traduction simultanée, thèse de Doctorat, ESIT, Université Paris III.

Gile, D. (1995a): Basic Concepts and Models for Interpreter and Translator Training, John Benjamins Publishing, Amsterdam.

GiLe, D. (1995b): Regards sur la recherche en interprétation de conférence, Presses universitaires de Lille. 
GiLe, D. (1985) : «Le modèle d'efforts et l'équilibre d'interprétation en interprétation simultanée», Meta 30-1, p. 44-48.

LAPLACE, C. (1998): «La traduction à vue dans la formation de l'interprète de conférence: principes théoriques et approche didactique», In TઐT, Terminologie et Traduction, la revue des services linguistiques des institutions européennes 2, p. 85-118.

Li, F. (2001): Etude de l'interprétation simultanée de conférence du chinois en français: spécificités linguistiques, solutions pratiques et retombées pédagogiques, thèse de Doctorat, université Paris III-Sorbonne Nouvelle.

Messina, A. (1998): “The Reading Aloud of English Language Texts in Simultaneously Interpreted Conferences", Interpreting 3-2, John Benjamins Publishing, p. 147-161

Newmark, P. (1988): Approaches to Translation, Hemel Hempstead, Prentice Hall.

OndeLLI, S. (1998): "Medium shifts in interpretation: Do interpreters produce oral texts?", The Interpreters' Newsletter 8, p. 181-193.

Pöchhacker, F. (1994): "Quality Assurance in Simultaneous Interpreting", In C. Dollerup and A. LindegaArd (Eds.), Teaching Translation and Interpreting 2: Insights, Aims, Visions, John Benjamins, Amsterdam.

Seleskovitch, D. et M. Lederer (1989): Pédagogie raisonnée de l'interprétation, Paris, Didier Erudition.

Weber, W. K. (1990): "The Importance of Sight Translation in an Interpreter Training Program", In D. M. Bowen (Eds.), Interpreting-Yesterday, Today, and Tomorrow, American Translators Association Scholarly Monograph Series IV, New York, State University of New York at Binghamton. 


\title{
Metacognitive Evaluation Method in Consecutive Interpretation for Novice Learners
}

\author{
JUNG YOON CHOI \\ ETI, University of Geneva, Geneva, Switzerland \\ pinkala@hanmail.net
}

\section{RÉSUMÉ}

Cet article est consacré à décrire la méthode d'évaluation métacognitive qui peut avoir une influence sur le processus d'enseignement et d'apprentissage des débutants en interprétation consécutive. L'idée fondamentale consiste à suggérer que les critères d'évaluation de l'interprétation des apprenants, surtout pour des débutants, devraient être différents de ceux de l'interprétation professionnelle. Le but, les problèmes d'évaluation d'un point de vue pédagogique et l'état de l'évaluation de l'interprétation en cours de formation sont abordés. Cet article joue un rôle primordial en évaluation étant donné qu'il cherche également à proposer un cadre pour l'évaluation de l'interprétation en classe et à présenter le concept de la courbe d'apprentissage, qui fait partie de l'évaluation du processus d'apprentissage. L'évaluation de l'interprétation et celle de la courbe d'apprentissage sont démontrées comme les deux piliers de la méthode de l'évaluation métacognitive.

\section{ABSTRACT}

This research mainly describes the metacognitive evaluation method that can affect both the teaching and learning process of learners, especially novice learners, in consecutive interpretation. The basic idea of this study is to argue that evaluation of novice learners should be based on standards differentiated from those geared towards professional interpreters. The purpose and limitations of evaluation from a pedagogical standpoint are examined, followed by an overview of evaluation in the interpretation classroom. This study is noteworthy in that it attempts to propose a framework for performance assessment and to introduce the learning curve concept as part of assessing the learning process, which are presented as the main elements of the metacognitive evaluation method.

\section{초록}

본 연구에서는 교육적인 관점에서 살펴본 평가의 목적과 문제점을 검토하고 통역 교육에서 결과 중심의 평가 방법을 재조명함으로써 순차통역을 처음 배우는 학습 자들의 교육 및 학습 과정을 반영하는 새로운 평가방식을 제시하는 데 그 목적을 두고 있다. 그런 의미에서, 현역 통역사들을 평가하는 잣대에 학습자들을 맞추는 것이 아니라, 학생들의 눈높이에 맞는 평가방식을 선보이고자 한다. 학습자들의 통 역실력뿐만 아니라, 자가 평가 능력을 함양하기 위해 실전 중심의 수행평가와 학습 과정 중심의 학습곡선 측정방식을 병행하는 초인지적 평가 방법을 제시하고 있다 는 데 주목할 필요가 있다.

\section{MOTS-CLÉS/KEYWORDS}

metacognitive evaluation method, performance assessment, learning process, learning curve assessment 


\section{Introduction}

It can be easily assumed that novice interpreters use different strategies from seasoned professionals. Moser-Mercer (1996: 52) argues that it is even more understandable for novice learners to use different strategies from intermediate or advanced learners. Kalina (1994: 229-231) also demonstrates that professionals face fewer interference problems and have lower correction rate for minor errors whereas students are overconcerned with even the slightest errors, thus sometimes negatively affecting the entire performance. According to Choi (2004), students will be judged by their performance as soon as they graduate and start to work in the interpretation market. But it is also a clear fact that students are not professionals. These would-be interpreters are in class not just to be judged by their end-product but also to learn. That is, to learn and practice until they get it right (Choi, 2004: 170).

Clear differences between students and professionals indicate a need to develop a different evaluation framework for students. A different framework that goes beyond judging students by their performance per se and branches out to equally assess the learning process is required. To that end, this study attempts to propose an outline for performance assessment and to introduce the learning curve concept as part of assessing the learning process. It should be borne in mind, however, that a more elaborate description of the performance assessment criteria and demonstration of the learning curve assessment criteria are not included in this article. Such issues will be elaborated in further studies in the future.

\section{Overview of evaluation and assessment in teaching}

\subsection{Definition and purpose of evaluation and assessment}

The term assessment has often been used interchangeably with the term evaluation. In fact, many of these terms are muddled in most people's minds including those engaged in teaching. According to Satterly (1989), assessment is a general term that includes all the processes and products that describe the extent and nature of the student's learning whereas evaluation means making value judgments of the effectiveness of teaching as a whole, which usually occurs after an assessment has been made (as cited in Child, 1973/2004: 361). According to Lefrançois (2000: 486-487), assessment is a term to appraise the student's performance and to judge the learning process whereas evaluation concerns making value judgments of the adequacy of teaching and the extent to which teaching goals have been met.

In short, it can be concluded that the main purpose of assessment is to measure the student's progress, to guide students and to examine the learning process whereas the purpose of evaluation is to judge the effectiveness of teaching, to see if the objective of teaching has been met and to inform both teachers and students of what is being expected.

\subsection{Types of assessment}

According to Child (1973/2004), four types of assessments are used depending on different purposes. They are pretask assessment, formative assessment, diagnostic assessment and summative assessment. First, pretask assessment aims at discovering 
the level of knowledge and skills of students before learning. It is often required that teachers, especially inexperienced teachers, devise a pretask assessment because it helps them have an idea about the level at which to pitch the course. Second, formative assessment, often used interchangeably with the term formative evaluation, is an on-going assessment method to assess the progress made in knowledge and skills during the learning process. In this type of assessment, the teacher's intention is to optimize feedback by making students aware of their weak and strong points, thereby guiding them to make improvements. Third, diagnostic assessment occurs particularly to pinpoint the cause of difficulties displayed by students who are struggling in a specific area during the learning process and to help them overcome such obstacles. This type of assessment mostly occurs during the formative assessment period. Child $(1973 ; 2004)$ also stresses that formative and diagnostic assessments are more processoriented approaches where students benefit the most since it provides feedback to remedy students' shortcomings and to reinforce their strengths. Fourth, summative assessment, also referred to as summative evaluation, normally occurs in the middle or at the end of a course or lesson to grade the students. Summative assessment, a more product-oriented approach, does not usually analyze difficulties nor provide subsequent feedback to the students but serves as useful information for students, teachers and employers to measure the student's learning results (Child, 1973; 2004: 361-363).

\subsection{Limitations}

During the evaluation process, many types of measuring instruments and methods are applied. Lefrançois (2000: 501) asserts that most of the problems relating to evaluation occur when these measuring instruments or methods fall short of two important standards, which are reliability and validity.

1) Reliability

According to Child $(1973 ; 2004)$, reliability in evaluation is often referred to as consistency as well. Child $(1973 ; 2004)$ points out that inconsistencies arise in one examiner or between several examiners over the same examinee due to fatigue, mood, different expectations and interpretations of the examinee's answers, experience or propensity of the examiners. To overcome such risks, Child $(1973 ; 2004)$ underlines the need for the examiners to coordinate the allocation and range of scores based on clearly defined criteria beforehand. Increasing the number of examiners or the length of examination, as mentioned by Child, can help enhance reliability as well. Examiners should also be cautious with borderline scores that should always be reexamined (Child, 1973; 2004: 368-370).

2) Validity

According to Child $(1973 ; 2004)$, validity is obtained when a test achieves what it has originally intended to achieve. Tests cannot be unreliable and valid but can be reliable and invalid since a reliable test can reveal unintended results (Child, 1973; 2004: 370-371). Lefrançois (2000) emphasizes that validity is most crucial especially for measuring instruments because test results would be of no use if a test fails to measure what it has intended 
to measure. In this context, educational or psychological tests are often challenged for their low validity deriving from unexpected variables (Lefrançois, 2000: 501).

\section{Evaluation in the interpretation classroom}

Choi (2003) describes what usually happens in a typical consecutive interpretation classroom in Korea. The student or the teacher improvises or reads the source text aloud. After the speech, one of the students is chosen to perform or voluntarily performs in front of the others. Students, then, provide oral feedback on their colleague's performance by indicating what went wrong content wise and expression wise, which is followed by the teacher's oral feedback (Choi, 2003: 223-224). In the interpretation classroom in Korea, most evaluation occurs through mid-terms, finals or during each class performance.

It is indispensable for would-be interpreters to be trained and evaluated in accordance with professional standards since they receive training in school to eventually work in the professional market. It is, therefore, natural for teaching to focus on interpretation skills, strategies and on how to teach them effectively. This enables students to live up to professional standards and to cope with problems that will occur in their career as interpreters. An extensive range of studies has already been conducted on what should constitute professional standards by Kurz (1993), Pöchhacker (1994) and Moser-Mercer (1996, 1998). In most cases, it would be plausible to say that multiple criteria components introduced in these studies can be explained by accuracy of meaning, appropriate expressions and presentation.

Choi $(1998,2004)$ describes some of the problems that interpreters encounter in the professional market, which concern terminology, preparation time, method, material and other variables. Regarding the tasks that an interpreter has to face in terms of preparation, she particularly classifies the preparation stage into three subcategories such as preparation of subject matter, terminology and last minute preparation. It has been demonstrated in her study (Choi, 2004) that one of the inevitable challenges that an interpreter has to cope with is the lack of preparation time and material especially during the busy conference season when the demand for interpretation reaches its peak and the interpreter is, thus, given so little time to prepare.

It is true that interpretation skills and strategies are of great significance in teaching. As a result, interpretation strategies and methods have been the bulk of studies pertaining to teaching interpretation. However, evaluating students entirely based on a yardstick geared towards professional standards runs the risk of defeating the very purpose of evaluation and assessment from a pedagogical standpoint. As mentioned before, the purpose of assessment is to measure the student's progress, to guide students and to examine the learning process while the purpose of evaluation is to judge the effectiveness of teaching and to see if the objective of teaching has been met.

Moser-Mercer (1996) and Gile (2001) have already reiterated the need to evaluate would-be interpreters differently. They both point out that assessment in the classroom has mostly been studied from the standpoint of professionals who usually judge performance on their self-established professional criteria or expectations of the actual users in the market. It is essential for assessment in the classroom to be judged from the standpoint of professionals since students should be aware of what is being expected from them in the professional market and to prepare themselves 
accordingly. It is, however, equally important for assessment to be studied from the student's standpoint as well. This is where student self-evaluation plays a vital role.

Evaluation does not only play a determinant role during the course of learning but it also plays an influential role in selecting students before they even start to learn. Moser-Mercer (1994: 65-66) suggests that though it is true that every school will have its own way of assessing a student's aptitude, a consensus can be reached on how to assess students since the ultimate goal shared by any training program is to develop students into competent professionals.

Metacognitive evaluation in this article, which will be discussed in 4., focuses on the implications of self-evaluation. The significance of self-evaluation in translation or interpretation classrooms has already been mentioned by Gile's (1993) processoriented approach, Sainz's (1993) student-focus process, Moser-Mercer's (1996) diarybased evaluation, Ficchi's (1999) self-directed learning and Lee-Jahnke's (2001) self-assessment. Despite the significance of self-evaluation displayed in previous studies, little study has been carried out to validate the hypothesis that self-evaluation can possibly lead to actual improvements in the student's learning process and performance in the area of interpretation.

\section{Metacognitive evaluation}

\subsection{Definition of metacognition}

Metacognition is described as thinking of thinking, knowing what we do and do not know or learning how to learn. Metacognitive strategies include talking about one's thinking, writing a thinking journal, self-evaluation and other strategies to solve problems that occur in the learning process (Blakey and Spence, 1990). The Oxford Dictionary of Psychology (2003) defines metacognition as follows;

Knowledge and beliefs about one's own cognitive processes [...] The term is also sometimes applied to regulation of cognitive functions including planning, checking or monitoring as one plans one's cognitive strategy for memorizing something, checks one's accuracy while performing mental arithmetic or monitors one's comprehension while reading $[\ldots]$

In a nutshell, metacognition in learning can be described as the awareness of the learning process and the ability to adapt to challenges that occur during this process through effective strategies, thereby helping learners improve their learning capacity.

\subsection{Purpose of metacognitive evaluation}

It is true that it is often challenging to discern whether the student has a problem in comprehension or in expression. Sometimes problems could concern both comprehension and expression. The student could have mistranslated a certain sentence due to shortcomings in processing the previous sentence. It is evident that many variables exist to explain the cause of poor performance. The purpose of metacognitive evaluation is to encourage students to think about such problems by reflecting upon themselves through self-evaluation. Through the Metacognitive Model, which will be illustrated later, students are taught to self-evaluate. Metacognitive evaluation also aims at assessing the learning results of self-evaluation to ultimately assess the learning process. 


\section{Metacognitive evaluation method}

\subsection{Performance assessment}

\subsubsection{Definition of performance assessment}

Lefrançois (2000: 488) describes performance assessment as an assessment that judges the actual performance of students in a teaching environment very similar and close to real life. Performance assessment is also referred to as authentic assessment, which Wiggins (1990) defines as directly judging student performance on worthy and practical intellectual tasks. It would not be an exaggeration to say that the bulk of evaluation in the interpretation classroom is based on performance assessments since most classes seek to simulate the actual situation that a professional will have to cope with in real life by often using authentic speeches, conducting mock conferences or providing students with first-hand experience to interpret real speakers themselves in school.

\subsubsection{Assessment method}

1) Assessment criteria

Performance assessment takes the form of summative assessment that occurs, as stated in 2.2., in the middle or at the end of a course or lesson to grade the students. To be able to assess performance, it is imperative to quantify performance on clearly defined criteria. As mentioned before, multiple criteria components in judging an interpreter's performance can boil down to accuracy of meaning, appropriate expressions and presentation. In this article, assessment on accuracy of meaning mostly focuses on omission, addition and mistranslation that occur between the source and target texts. Appropriate expressions concerns grammar, terminology in the target text. Presentation can consist of multiple sub-parameters such as voice, speed, articulation, and rhythm, to mention a few.

Considering that the aforementioned criteria are still far from sufficient, further study will be carried out to specify the categories that constitute the performance assessment criteria.

2) Rating scale

It is true that rating performance has been highly disputed due to its subjective nature. Teachers often have to make sure that evaluation is valid and reliable, which has been the major challenge in evaluation as mentioned in 2.3. Quantifying performance quality can somewhat minimize the risks that are inherent in assessments by making performance quality more measurable. The five-point Likert-type rating scale (as cited in Christiansen, 2005), commonly used as a data gathering tool in psychology and teaching, is employed as an assessment tool to quantify performance. Accuracy, expressions and presentation, the main elements of the performance assessment criteria, are scored respectively. For measurement purposes, each rating is assigned with points ranging from 6 to 10. For instance, if a student performance is excellent in accuracy, good in expressions and acceptable in presentation, the student receives 10 points for accuracy, 9 points for expressions and 8 for presentation. The rating scale will also be further elaborated in future studies. Meanwhile, an example of the rating scale sheet is illustrated in Table 1. 
TABLE 1

Five-point scale based on the Likert-type rating scale

\begin{tabular}{|l|c|c|c|c|c|}
\hline Rating & Inferior & Poor & Acceptable & Good & Excellent \\
\hline Points & 6 & 7 & 8 & 9 & 10 \\
\hline
\end{tabular}

3) Assessment criteria composition

After having been scored respectively through the five-point scale, the three categories are also given different weight value. Accuracy of meaning can be assumed to be the most determinant factor, followed by expressions and presentation when assessing student performance since students, particularly novice students, should be carefully guided to concentrate their efforts more on grasping the accurate sense than on demonstrating flowery expressions and superior presentation skills. Therefore, accuracy is multiplied by a factor of 5 and expression by a factor of 3 . Presentation is multiplied by the lowest factor of 2 to make sure that nobody with excellent presentation skills will be able to compensate for poor accuracy. Following is a sample of the performance assessment sheet based on the aforementioned criteria and the rating scale.

TABLE 2

Performance assessment sheet

\begin{tabular}{|l|l|l|l|}
\hline & Points & Weight & Weighted score \\
\hline Accuracy & & 5 & Points $\times 5=$ \\
\hline Expressions & & 3 & Points $\times 3=$ \\
\hline Presentation & & 2 & Points $\times 2=$ \\
\hline Composite score & $/ 100$ & \\
\hline
\end{tabular}

\subsection{Learning curve assessment}

\subsubsection{Definition of learning curve}

According to the Oxford Dictionary of Psychology (2003),

Learning curve is a mathematical expression of the change in behavior occurring as a function of practice, usually represented by a graph showing a measure of performance on the vertical axis and amount of learning represented by trials or time on the horizontal axis [...] The concept was introduced by the US psychologist Louis Leon Thurstone $[\ldots]$

Constructing a learning curve could enable both teachers and students to better understand the learning process. In his study, Thurstone (1919) states that the level of attainment obtained per unit of practice decreases as practice increases. He admits that this tendency, called the law of diminishing returns, may not necessarily apply to the learning of foreign languages and other complex processes of learning where learning curve positively accelerates in the initial stage, remains steady during the course of learning with irregular changes from time to time. His study on learning curves advocates that continued learning in some fields may not demonstrate a rise of the practice limit where attainment stabilizes at a certain level. However, that limit of practice in the initial stage of learning can be, instead, achieved at a faster rate or with more consistency in learning (Thurstone, 1919:11-12). Atherton describes the 
learning curve as the amount learned against the number of trials or over time. Atherton also refers to consistency as the most vital factor upon which the progress of learning is assessed since novice learners can get the performance right by chance, which people call "beginner's luck" (Atherton, 2003).

\subsubsection{Assessment method}

1) Five-Stage Metacognitive Model

Learning curve assessment is mainly based on the following Five-Stage Metacognitive Model.

Five-Stage Metacognitive Model (Choi, 2004:181)

1) Stage 1: self-evaluation $\left(\mathrm{S}^{1}\right) /$ feedback $\left(\mathrm{T}^{2}\right)$

2) Stage 2: problem-finding $(\mathrm{S}, \mathrm{T}) /$ student profiling $(\mathrm{T})$

3) Stage 3: prioritization $(\mathrm{S}, \mathrm{T})$

4) Stage 4: practice (S)

5) Stage 5: revaluation (S) / monitor $(\mathrm{T})$

According to Choi (2004), in stage 1, students record their performance in class and listen to it after class. They submit their tapes and self-evaluated texts to the teacher. The teacher listens to the tapes again and provides additional feedback. In stage 2, the student and teacher identify the root causes of the student's poor performance together, whether it be comprehension, expression, speed, note-taking skills, lack of practice or other shortcomings. As pointed out by Lim (2001: 220), one of the problems students, particularly novice students, often face is that they have a tendency to want to cling to words and fail to disassociate the words from the idea. In parallel with the problem-finding process, the teacher develops the student's individual file, what is referred to as a student portfolio by Lefrançois (2000: 487), containing the samples of their self-evaluated texts, their fundamental weaknesses, strengths, habits, personality traits, anything that the teacher believes is worthwhile to keep track of the student's learning process. In stage 3, students prioritize the problems with the help of the teacher when multiple problems are interwoven since they cannot address them all at once. In stage 4 , students concentrate their practice on the most critical issue based on the assessment made in stage 1, 2 and 3. In stage 5, students revaluate themselves while the teacher continues to monitor them on a regular basis (Choi, 2004: 182).

One of the weaknesses of this method, however, is that student performance is only audio-taped and not video-taped. For technical reasons, visual presentation skills such as eye contact and gestures, which are critical factors in consecutive interpretation, are not measured in performance assessment. However, they are dealt with in class to make sure that students do not disregard the significance of these skills.

\section{2) Assessment procedure}

A pretask assessment is carried out at the beginning of the course before they embark on learning. First-time learners in consecutive interpretation undergo the same memory test from their B language into A. If a class consists of second-time learners who have already learned consecutive interpretation in the previous semester, students will take a simple consecutive interpretation test. This is essential for the teacher to obtain information about the student's outset level. Learning curve assessment also takes the form of formative and diagnostic assessments since the student is subject to 
the metacognitive model that enables both the teacher and the student to detect the student's weaknesses, strengths and causes of poor performance. Summative assessment is also employed since the results of the mid-terms and finals are used to compare the degree of progress made between the pretask assessment, mid-term and final examinations.

While performance assessment concentrates on the results of performance, learning curve assessment puts more focus on the degree of progress made in performance during the course of learning. Other variables including self-evaluation capabilities may well play a significant role in assessing the learning patterns of individuals, which will determine the learning curve assessment criteria. Such issues will be specified in future studies.

\section{The Metacognitive Evaluation Method}

Based on what has been discussed above, the Metacognitive Evaluation Method can be summarized as follows:

TABLE 3

The Metacognitive Evaluation Method

\begin{tabular}{|l|l|l|l|}
\hline \multirow{3}{*}{ Purpose } & \multicolumn{3}{|l|}{$\begin{array}{l}\text { Tetacognitive Evaluation } \\
\text { effectiveness in the process and results of learning }\end{array}$} \\
\hline Method & & Performance assessment & Learning curve assessment \\
\cline { 2 - 4 } & Purpose & $\begin{array}{l}\text { Quantify performance close to } \\
\text { real-life and use it as a tool to } \\
\text { assess the learning progress }\end{array}$ & $\begin{array}{l}\text { Quantify the learning progress } \\
\text { of consecutive interpretation, } \\
\text { self-evaluation and other param- } \\
\text { eters necessary to assess the } \\
\text { learning progress }\end{array}$ \\
\cline { 2 - 4 } & Tool & Weighted rating scale & Five-Stage Metacognitive Model \\
\cline { 2 - 4 } & Type & Summative & $\begin{array}{l}\text { Pretask, formative, diagnostic, } \\
\text { summative }\end{array}$ \\
\hline
\end{tabular}

\section{Conclusion and discussions}

The purpose and method of metacognitive evaluation in the consecutive interpretation classroom have been discussed. On-going study, however, is required to elaborate the performance and learning curve assessments criteria. Empirical study based on research updates on the actual results of performance and learning curve assessments are also needed to support their criteria. The learning curve assessment criteria, in particular, needs to be specified through thorough and careful investigation to demonstrate the student's learning process and to go as far as to translate qualitative data into a subsequent learning curve. This is indispensable to validate the hypothesis that the learning curve assessment in parallel with the performance assessment mechanism under the Metacognitive Evaluation Method can serve as a moderate step towards the development of a different method to assess students in the classroom and perhaps carefully predict their potential to make further advance in their career as professional interpreters. 


\section{NOTES}

1. Student.

2. Teacher.

\section{REFERENCES}

Atherton, J-S. (2003): Learning and Teaching: Learning Curve [On-line], Retrieved January 25, 2005, <http://www.learningandteaching.info/learning/lerncrv.htm>.

Blakey, E and S. SPence (1990): Developing Metacognition. ERIC Digest, ERIC Clearinghouse on Information Resources Syracuse NY, Retrieved April 7, 2005, <http://www.ericdigests.org/ pre-9218/developing.htm>.

Child, D. (1973/2004): Psychology and the Teacher, London/New York, Continuum, p. 359-389.

Cног, J.W. (1998): Tongyeok-beonyeok-ipmun [Introduction to Interpretation and Translation], Seoul, Sinron Publishing Co.

Cног, J.W. (2004): "Preparing for an International Conference," Conference Interpretation and Translation 6-2, p. 187-200.

Сног, J-Y. (2003): “The interpretive feedback model," Conference Interpretation and Translation 5-2, p. 221-234.

Сног, J-Y. (2004): “A Metacognitive Approach to Evaluating Consecutive Interpretation for Novice Learners," Conference Interpretation and Translation 6-2, p. 169-184.

Christiansen, J. (n.d.): Examples of Likert-type Responses Sometimes Used in Data Gathering Instruments, Retrieved April 3, 2005, <http://extensioneducation.tamu.edu/Teaching/likertscale_2.htm>

Colman, A-M. (2003): Oxford Dictionary of Psychology, Oxford/New York, Oxford University Press.

Fichнi, V. (1999): “Learning Consecutive Interpretation,” Interpreting 4-2, p. 204-214.

GiLE, D. (1993): “The Process-oriented Approach in Translation Training," Teaching Translation and Interpreting 2, p. 107-112.

GiLe, D. (2001): "L'évaluation de la qualité de l'interprétation en cours de formation», Meta 46-2, p. 379-392.

Kalina, S. (1994): “Analyzing interpreters' performance: Methods and problems," In C. Dollerup and A. Lindegand (Eds). Teaching Translation and Interpreting 2: Insights, Aims, Visions, Amsterdam/Philadelphia, John Benjamins.

Kurz, I. (1993): "Conference Interpretation: Expectations of Different User Groups," In the Interpreters' Newsletter 5, Università degli Studi di Trieste.

Lee-JahnKe, H. (2001): “Aspect pédagogique de l'évaluation en traduction, Meta 46-2, p. 259267.

Lefrançois, G-R. (2000): Psychology for Teaching (10 ${ }^{\text {th }}$ ed), Wadsworth.

Lim, H-O. (2001): "Teaching Interpretation and Translation," Conference Interpretation and Translation 3, p. 211-242.

Moser-Mercer, B. (1994): "Aptitude Testing for Conference Interpreting: Why, When and How," In S. Lambert and B. Moser-Mercer (Eds.), Bridging the Gap: Empirical Research in Simultaneous Interpretation, Amsterdam/Philadelphia, John Benjamins.

Moser-Mercer, B. (1996): "Quality in Interpreting some Methodological Issues," In The Interpreters' Newsletter 7, Università degli Studi di Trieste.

Moser-Mercer, B. (1998): “Measuring Quality in Interpreting," In Ocena tlumaczenia ustnego: Evaluating an Interpreter's Performance, Lodz.

Pöchhacker, F. (1994): “Quality Assurance in Simultaneous Interpreting," In C. Dollerup and A. LindegaARD, Teaching Translation and Interpreting 2: Insights, Aims, Visions, Amsterdam/ Philadelphia, John Benjamins.

SAinz, M.-J. (1993): "Student-centered Corrections of Translations," Teaching Translation and Interpreting 2, p. 135-141. 
Thurstone, L.-L. (1919): “The Learning Curve Equation,” In J.-R., Angell, H.-C. Warren, S.-I. Franz and M. Bentley (Eds). Psychological Monographs 26-3, Princeton, N.J./Lancaster, PA.

Wiggins, G. (1990): "The Case for Authentic Assessment," Practical Assessment, Research and Evaluation 2-2, Retrieved April 10, 2005, <http://PAREonline.net/getvn.asp?v=2\&n=2>. 